Article

\title{
Cutibacterium acnes Biofilm Study during Bone Cells Interaction
}

\author{
Céline Mongaret ${ }^{1,2, * \mathbb{D}}$, Jennifer Varin-Simon ${ }^{1} \mathbb{D}$, Fabien Lamret ${ }^{1} \mathbb{D}$, Taghrid S. El-Mahdy ${ }^{1,3,4}$, \\ Lucien Brasme ${ }^{5}$, Véronique Vernet-Garnier ${ }^{5}$, Sophie C. Gangloff ${ }^{1} \mathbb{D}$, Xavier Ohl ${ }^{1,6}$ and \\ Fany Reffuveille ${ }^{1}$ (D)
}

1 EA 4691 Biomatériaux et Inflammation en Site Osseux (BIOS), Université de Reims Champagne-Ardenne, SFR Cap Santé (FED 4231), 51100 Reims, France; jennifer.varin-simon@univ-reims.fr (J.V.-S.); fabien.lamret@univ-reims.fr (F.L.); sata186@hotmail.com (T.S.E.-M.); sophie.gangloff@univ-reims.fr (S.C.G.); xohl@chu-reims.fr (X.O.); fany.reffuveille@univ-reims.fr (F.R.)

2 Service Pharmacie, Centre Hospitalier Universitaire de Reims (CHU Reims), 51100 Reims, France

3 Department of Microbiology and Immunology, Faculty of Pharmacy, Helwan University, Cairo 11795, Egypt

4 Department of Microbiology, Faculty of Pharmacy, Ahram Canadian University, Cairo 12585, Egypt

5 Laboratoire de Bactériologie-Hygiène, Centre Hospitalier Universitaire de Reims (CHU Reims), 51100 Reims, France; lbrasme@chu-reims.fr (L.B.); vvernetgarnier@chu-reims.fr (V.V.-G.)

6 Service d'Orthopédie et Traumatologie, Centre Hospitalier Universitaire de Reims (CHU Reims), 51100 Reims, France

* Correspondence: celine.mongaret@univ-reims.fr

Received: 30 July 2020; Accepted: 10 September 2020; Published: 12 September 2020

check for updates

\begin{abstract}
Cutibacterium acnes is an opportunistic pathogen involved in Bone and Prosthesis Infections (BPIs). In this study, we observed the behavior of commensal and BPI C. acnes strains in the bone environment through bacterial internalization by osteoblast-like cells and biofilm formation. For the commensal strains, less than $1 \%$ of the bacteria were internalized; among them, about $32.7 \pm 3.9 \%$ persisted intracellularly for up to $48 \mathrm{~h}$. C. acnes infection seems to have no cytotoxic effect on bone cells as detected by LDH assay. Interestingly, commensal C. acnes showed a significant increase in biofilm formation after osteoblast-like internalization for $50 \%$ of the strains (2.8-fold increase). This phenomenon is exacerbated on a titanium support, a material used for medical devices. For the BPI clinical strains, we did not notice any increase in biofilm formation after internalization despite a similar internalization rate by the osteoblast-like cells. Furthermore, fluorescent staining revealed more live bacteria within the biofilm after osteoblast-like cell interaction, for all strains (BPIs and commensal). The genomic study did not reveal any link between their clinical origin and phylotype. In conclusion, we have shown for the first time the possible influence of internalization by osteoblast-like cells on commensal C. acnes.
\end{abstract}

Keywords: joint infections; biofilms; host-pathogen interactions; Cutibacterium acnes; internalization

\section{Introduction}

Cutibacterium acnes is a commensal bacterium playing an important role in the ecosystem of healthy human skin. However, this species is also recognized as a pathogen in foreign body infection: endocarditis, prostatitis and specifically in Bone and Prosthesis Infections (BPIs) [1], for which C. acnes has an incidence of approximately $10 \%$ (more frequently in the shoulder, the hip, the spine and the knee joints) $[2,3]$. C. acnes strains responsible for BPIs mostly originate from the patient's own normal skin microbiota [4]. C. acnes is still isolated after surgery despite skin disinfection [5]. The hypothesis is that $C$. acnes hide in the hair bulbs and are spilled during surgical incision. 
C. acnes infections do not present obvious clinical symptoms, which appear more than 3 months after surgery $[1,2]$. Low-grade clinical symptoms and specific bacteriological culture conditions, a prolonged incubation time in anaerobic conditions of growth, demonstrate that its clinical diagnosis is challenging. Indeed, C. acnes infections do not induce a clear inflammatory response, meaning this microorganism is able to escape the immune system, probably thanks to the bacterial internalization by host cells [6]. Aubin et al. revealed that $C$. acnes isolates from BPIs were internalized by human osteoblast-like cells [7]. These hidden bacteria in osteoblast-like cells could explain the chronicity of BPIs, an event already described by Josse et al. with Staphylococcus aureus, species mainly involved in BPIs. The internalization by osteoblasts is a key element to protect $S$. aureus from the immune system and to sustain the infection [8]. From this observation, the internalization by osteoblast-like cells could support the hypothesis of an increased resistance of $C$. acnes to immune system attacks or antibiotics [8].

C. acnes infections are difficult to treat, as this anaerobic bacillus is able to form biofilms on surgical material because the host rapidly coats the surface of an implant with extracellular matrix proteins on which bacteria can easily adhere, which is the first step of biofilm formation [9]. The main problem of $C$. acnes biofilm is that the embedded bacteria are less susceptible to antimicrobials than their planktonic counterparts as the biofilm is a complex bacterial community with altered phenotypes surrounded by a protective matrix composed of extracellular DNA, protein and polysaccharides [10]. Moreover, the $C$. acnes biofilm formation also increases the putative virulence factor expression $[6,11]$. Holmberg et al. observed that isolates from deep infections produced more biofilm than skin isolates, highlighting the role of biofilms in C. acnes virulence, regardless of clinical origin [12].

Indeed, C. acnes could be classified by its clinical origins, its virulence phenotype and also by its genomic evolution. C. acnes is subdivided into six main phylotypes: IA1, IA2, IB, IC, II and III. Mac Dowel et al. has correlated several phylotypes of $C$. acnes with several clinical pathways [13]. Indeed the various subgroups play a significant role in different skin disorders [14]. For example, Paugam et al. demonstrated that phylotype IA1 is predominant in acne lesions in clinical dermatology, a result found after performing a prospective observational study [15]. More recently, Kilian et al. have highlighted the presence of subgroups in the phylotypes called multilocus sequence typing (MLST), according to a genome analysis [16]. Using different lineages of $C$. acnes, Aubin et al. investigated the MLST profile and $C$. acnes host-pathogen interaction in BPIs, identifying two $C$. acnes behaviors depending on their genetic background clone complex (CC), although these groups are not correlated to clinical origin [7]. A correlation between genomic (MLST profile) and proteomic (SELDI-TOF profile) data was previously described by Dekio et al., suggesting that the microenvironment of each $C$. acnes subtype may influence protein expression [17]. All these previous studies suggest that $C$. acnes virulence behavior is linked to the clinical origin or environment without any correlation with genetic background.

In this study, we hypothesized that bone environment, especially internalization by host cells, could induce $C$. acnes pathogenesis. We analyzed the behavior of clinical commensal $C$. acnes isolates and then compared them to the BPI isolates before and after internalization by osteoblast-like cells. Finally, we investigated their phylotype and CC profile to identify a potential link between genetic background and virulence behaviors.

\section{Materials and Methods}

\subsection{Culture Media}

The human osteosarcoma cell line SaOS2 purchased from ATCC (ATCC ${ }^{\circledR}$ HTB $85^{\mathrm{TM}}$ ) was cultured in a $5 \% \mathrm{CO} 2$ atmosphere at $37^{\circ} \mathrm{C}$ in Dulbeccco's Modified Eagle Medium (DMEM, Gibco, Invitrogen, Carlsbad, CA, USA) supplemented with $10 \%$ of fetal bovine serum (Gibco, Invitrogen, Carlsbad, CA, USA) and a $\%$ antibiotic PenStrep solution (Gibco, Invitrogen, Carlsbad, CA, USA). The medium was changed every two days. 


\subsection{Bacteria Strains Culture}

Cutibacterium acnes (formerly Propionibacterium acnes) strains were isolated and anonymized at the laboratory of bacteriology of Reims University hospital (CHU Reims). Fifteen strains were isolated by swabbing from fifteen different patients: nine BPIs and six commensal isolates. C. acnes BPIs were defined when at least three of the five samples from the bone and joint tissue during orthopedic surgery were positive. The positivity was discussed and confirmed by the consultation of an expert multidisciplinary team (named CRIOA: national and referent center for bone and joint infections). When less than three $C$. acnes positive samples were identified from the samples, which were not from bone and joint tissue, the strains were defined as commensal strains. Implant-associated infections were confirmed using the Infectious Diseases Society of America criteria for bone and joint infections [16]. The clinical source of the isolates is mentioned in Table 1. The reference strain ATCC 6919 was isolated from facial acne and used as the referent strain to identify the variability of the $C$. acnes virulence strains with a different clinical origin. C. acnes strains were isolated on Columbia agar with $5 \%$ sheep blood (BioRad, Hercules, CA, USA) and cultivated in a Brain Heart Infusion (BHI) broth (BioRad, Hercules, CA, USA) for five days under anaerobic conditions using the GenBox system (Biomerieux, Marcy $1^{\prime}$ Etoile, France) at $37^{\circ} \mathrm{C}$.

Table 1. Phylotype and multilocus sequence typing (MLST) profiles of the C. acnes commensal and Bone and Prosthesis Infection (BPI) pathogenic strains.

\begin{tabular}{ccccc}
\hline Isolate Name & Clinical Source & Sequence Typing & Clonal Complex MLST & Phylotype \\
\hline C2 & Skin & 107 & CC107 & IC \\
\hline C5 & Skin & 1 & CC1 & IA1 \\
\hline C7 & Skin & 49 & singleton & IA1 \\
\hline C9 & Skin & 95 & singleton & IA1 \\
\hline C18 & Skin & 46 & CC1 & IA1 \\
\hline BPI1 & $\begin{array}{c}\text { Shoulder } \\
\text { prosthesis }\end{array}$ & 20 & CC1 & IA1 \\
\hline BPI2 & $\begin{array}{c}\text { Shoulder } \\
\text { prosthesis }\end{array}$ & 1 & CC1 & IA1 \\
\hline BPI3 & $\begin{array}{c}\text { Shoulder } \\
\text { prosthesis }\end{array}$ & 20 & CC1 & IA1 \\
\hline BPI4 & Spine material & 21 & CC4 & IA1 \\
\hline BPI5 & Spine material & 1 & SC1 & IA1 \\
\hline BPI6 & $\begin{array}{c}\text { Shoulder } \\
\text { prosthesis }\end{array}$ & 51 & CC4 & IB \\
\hline BPI7 & Hip prosthesis & 55 & CC5 & IA1 \\
\hline BPI8 & $\begin{array}{c}\text { Shoulder } \\
\text { prosthesis }\end{array}$ & 152 & CC5 & IB \\
\hline BPI9 & Tibia material & 152 & IB \\
\hline
\end{tabular}

\subsection{C. acnes Internalization by Osteoblast-Like Cells}

Bacterial internalization experiments were adapted from Josse et al.'s study [18]. Briefly, $10.5 \times 10^{3} \mathrm{SaOS} 2$ cells $/ \mathrm{cm}^{2}$ were seeded in 24-well culture plates and were incubated at $37^{\circ} \mathrm{C}$ for $72 \mathrm{~h}$. Cell cultures were then washed with a phosphate-buffered saline solution (PBS, Gibco, CA, USA) and incubated overnight with $1 \mathrm{~mL}$ of media without antibiotics. The following day, cells were washed with PBS and incubated with $1 \mathrm{~mL}$ of media without antibiotics. One well was used for counting the number of cells per well. Bacteria were centrifuged for $5 \mathrm{~min}$. at $5000 \times g$, and the pellets were rinsed with PBS. Bacteria were then added to the culture medium to obtain a multiplicity of infection (MOI) of 100:1 (C. acnes: cell). After $3 \mathrm{~h}$ of interaction, the cells were washed with PBS and 
incubated with cell medium containing $100 \mu \mathrm{g} / \mathrm{mL}$ of gentamicin (Fisher Scientific, Hampton, $\mathrm{NH}$, USA), during $1 \mathrm{~h}$ at $37^{\circ} \mathrm{C}$, in a $5 \% \mathrm{CO} 2$ humidified atmosphere. Then, cells were washed again with PBS and a 0.1\% Triton X-100 (Sigma, Saint Louis, MO, USA) solution was added to each well to harvest the intracellular bacteria. Lysates were seeded on blood agar plates using easySpiral (Intersciences, St Nom de la Breteche, France) at $37^{\circ} \mathrm{C}$ under anaerobic condition, using the GenBag system for 5 days to determinate the number of recovered colony-forming units (CFU). The percentage of bacteria was calculated as follows: \% of bacteria $=(\mathrm{CFU} / \mathrm{mL} \times 100) /($ number of cells $\times \mathrm{MOI})$. Each experiment was done three (independent) times and with at least two technical repeats (at least six raw data points were used in the statistical analysis).

\subsection{LDH Measurement Assay}

To determine the bacterial impact on the osteoblast-like cells, the lactate dehydrogenase (LDH) activity was evaluated with a cytotoxicity detection kit (Roche, Bâle, Switzerland), following the manufacturer's instruction. Briefly, after $3 \mathrm{~h}$ of cells/bacteria interaction and after $48 \mathrm{~h}$ post-infection, supernatants were collected and filtered to remove cells debris and bacteria. Then, they were incubated with a substrate during $15 \mathrm{~min}$ in the dark and absorbance was measured at $490 \mathrm{~nm}$ with a correction at $700 \mathrm{~nm}$. Each experiment was done three (independent) times and with at least two technical repeats (at least six raw data points).

\subsection{Static Biofilm Model (Crystal Violet Staining Model)}

As previously described, the biofilm biomass was evaluated by crystal violet staining $[19,20]$. An isolated colony of $C$. acnes was diluted in $1 \mathrm{~mL}$ of BHI medium in a 48-well plastic microtiter plate or in presence of a titanium piece. After 5 days of anaerobic incubation at $37^{\circ} \mathrm{C}$, the planktonic growth was evaluated by measuring the absorbance at $600 \mathrm{~nm}$. The plates were gently washed 3 times with water and $1 \mathrm{~mL}$ of $0.18 \%$ crystal violet was applied for $20 \mathrm{~min}$. After washing with water, $1 \mathrm{~mL}$ of $95 \%$ ethanol was added to each well and thus the stained biofilm was evaluated by measuring the absorbance at $595 \mathrm{~nm}$. All results are expressed with the subtraction of the blank: medium without bacteria. Each experiment was done three (independent) times and with at least three technical repeats (at least nine raw data points).

\subsection{Fluorescent Microscopy}

Biofilms were stained using the LIVE/DEAD BacLight Bacterial Viability kit (Molecular Probes, Eugene, OR) for the microscopy experiments. A ratio of SYTO-9 (green fluorescence, all live and dead cells) to propidium iodide (PI) (red fluorescence, damaged bacterial membrane cells or "dead" bacteria) of 1:5 was used. Image acquisitions were performed on an Axiovert 200M inverted microscope using a $\times 40$ objective and the dedicated Axiovision v 3.2.6 software (Carl Zeiss, Oberkochen, Germany). Equal acquisition times were set for each SYTO-9 and PI channel in any condition of all the experiments. Surface quantification of live and dead bacteria was determined using ImageJ software (v1.50i, National Institutes of Health, Bethesda, MD, USA). Each experiment was done three (independent) times and with at least two technical repeats (at least six raw data points).

\subsection{Persistence of Biofilm}

After internalization, bacteria were seeded at different passages on blood agar plates or on a BHI solution to observe the persistence of the biofilm. From Culture 1 (P1 = bacteria seeded after $3 \mathrm{~h}$ and Gentamicin treatment after internalization) to Culture 5, PO represents the bacteria culture before internalization. Biofilm formation was measured by crystal violet staining as described above. Each experiment was done three (independent) times and with at least three technical repeats (at least nine raw data points). 


\subsection{Phylotype and MLST Data Analysis}

Phylotype and MLST were performed according to the protocol available at https://pubmlst. org/pacnes/ and the clonality was carried out by MLST on all isolates as previously described [20]. Briefly, bacterial DNA was extracted using the QIAmp DNA Mini kit (Qiagen, Hilden, Germany) according to the manufacturer's instruction. Bacterial genes $\operatorname{aroE}, \operatorname{atpD}, \operatorname{guaA}, \operatorname{lep} A, \operatorname{sod} A$, tly and camp2 were amplified by end-point PCR. DNA amplification samples and sequencing primers were sent to the Genewiz society for sequencing bacteria. For each gene, the DNA sequences were entered into the "Cutibacterium acnes MLST database" to identify the corresponding allele. All gene alleles were recorded in the database and, for each C. acnes strain, a phylotype and MLST profile were automatically attributed by the pubmlst database.

\subsection{Statistical Methods}

All values are the means of at least three independent experiments (three biological repeats). Moreover, each experiment was done at least in two (internalization experiment) or three (crystal violet model) technical repeats (at least six to nine raw data points). The statistical significance of the results was assessed using non-parametric analysis with pairwise tests. The exact non-parametric Wilcoxon-Mann-Whitney test for independent samples was used (StatXact 7.0, Cytel Inc). Non-parametric statistics were used owing to the lack of a normal distribution of the assessed variables. Stratification allowed the impact of technical variability to be taken into account. Differences were considered significant at $p<0.05$.

\section{Results}

\subsection{Commensal C. acnes Strain Behaviors}

C. acnes strains isolated from patients who did not declare $C$. acnes infection (C "commensal" strains) were studied for their capacity to form biofilms: five strains belonged to the IAI phylotype and one to the IC phylotype, according to the MLST assay (Table 1).

The crystal violet staining assay allowed quantifying the $C$. acnes biofilm biomass. After 5 days of biofilm development, we observed that the six commensal strains formed low amounts of biofilm with an average absorbance of $0.13 \pm 0.02$ for the $C$ strains (from a value of 0.06 for $C 2$ to a value of 0.25 for C8; Figure 1a), also noticed for the lab strain ATCC 6919.

(a)

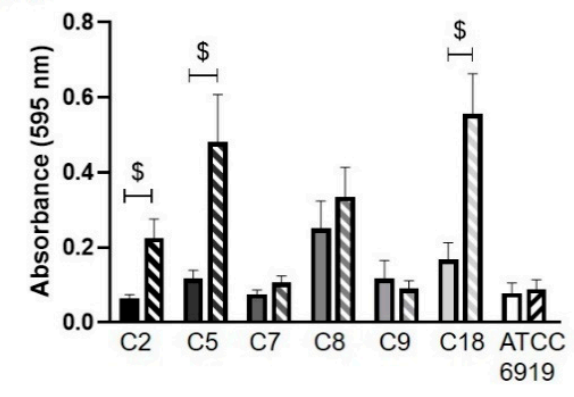

(b)

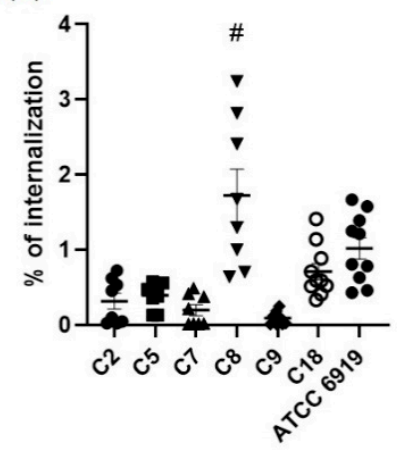

Figure 1. Behaviors of the commensal C. acnes strains. (a) Biofilm forming capacity by crystal violet quantification before (full bars) and after internalization (hatched bars) by osteoblast-like cells ( $\$ ; p<0.05$ compared to initial biofilm quantification); (b) internalization rate (\%) by osteoblast-like cells (\#; $p<0.05$; different to all other strains).

We have not noticed a significant difference in planktonic growth for all the tested strains before and after internalization by osteoblast-like cells (Figure S1). 
Then, we evaluated the rate of internalization after $3 \mathrm{~h}$ of co-culture of these strains by osteoblast-like cells (SaOS2). The mean percentage of internalized commensal bacteria by osteoblast-like cells was $0.58 \pm 0.1 \%$ (Figure 1b). Strain C 8 had an internalization rate of $1.72 \pm 0.35 \%$, which was significantly higher and more variable than the other strains. As observed through LDH assay quantification, the interaction between $C$. acnes and osteoblast-like cells for three hours had no consequence on cell cytotoxicity (Figure S2).

To determine if the internalization could have a specific impact on C. acnes capacity to form a biofilm, internalized bacteria were collected and cultured to monitor the biofilm formation after 5 days of incubation. We observed an average absorbance of $0.368 \pm 0.04$ for all commensal strains, from 0.09 for $\mathrm{C} 9$ to 0.56 for strain $\mathrm{C} 18$, which possessed the highest biofilm coloration. Furthermore, comparing the quantity of formed biofilm by C. acnes before (Figure 1a) and after the internalization (Figure 1a), a significant increase in biofilm formation was revealed after internalization for three C. acnes commensal strains, C2, C5 and C18, (with fold-increase rates of 3.83, 4 and 3.3; respectively) and a non-significant increase for one strain (C8 with a fold-increase of 1.4).

To deepen this approach, using live/dead fluorochromes, the percentage of Syto9 and PI (damaged bacterial membrane or "dead" bacteria) staining was determined in the fluorescent images of the initial and post-internalization biofilm of the commensal strains (Figure 2). We observed a mean proportion of $50.4 \%$ of live bacteria before internalization (values from 16 to $72 \%$ according to the strains), which increased to $80.6 \%$ after internalization ( $p<0.05$; values from 58 to $97 \%$ ).

(a)

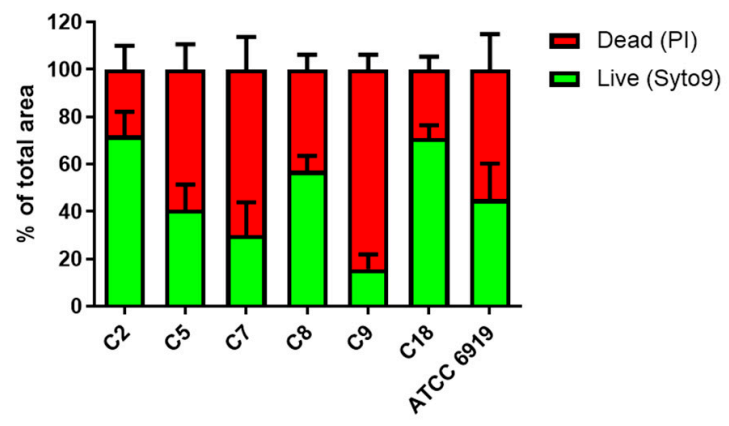

(b)

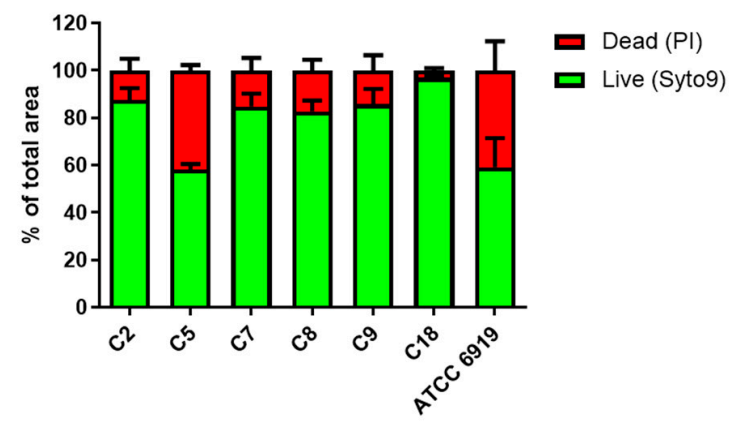

Figure 2. The living bacterial proportion in the commensal C. acnes strain biofilms. Repartition of Syto9 (green bars) and PI (red bars) staining within biofilms formed by the commensal C. acnes strains before (a) and after internalization (b) by osteoblast-like cells. Acquisition of images by fluorescent microscopy and calculation by Image J software.

\subsection{Commensal C. acnes Fate after Internalization}

The stability of the behavioral change after internalization for the three commensal C. acnes strains with significant modification ( $\mathrm{C} 2, \mathrm{C} 5$ and $\mathrm{C} 18)$ and the $\mathrm{C} 8$ strain, which possessed a tendency for a similar behavior, was tested by performing subcultures of these strains and by quantifying the formed biofilm after each culture (Figure 3a). We observed a stable increased biofilm amount for 2 of the 4 strains ( $\mathrm{C} 2$ and $\mathrm{C} 5$ ), underlining a change in bacterial metabolism. After a $3 \mathrm{~h}$ interaction and antibiotic treatment, we enumerated the quantity of the intracellular and extracellular bacteria after $48 \mathrm{~h}$ of co-culture to investigate the possibility of persistence of the C. acnes strains in osteoblast-like cells (Figure 3b).

We observed that a low percentage of initial number of bacteria quantified as intracellular after $3 \mathrm{~h}$ of interaction were externalized (3.9 $\pm 3 \%$ (C8) to $17.2 \pm 9.6 \%(\mathrm{C} 2))$. The majority of the bacteria were either still in the osteoblast-like cells $(32.7 \pm 3.9 \%$ ) or supposedly died ( $>50 \%)$ (Figure $3 \mathrm{~b}$ ). Furthermore, we tested the cytotoxicity effect of the internalized bacteria on the osteoblast-like cells. No difference in LDH measurement was observed between infected and non-infected cells (Figure 3c). 
(a)

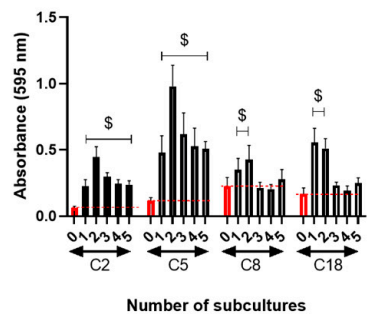

(b)

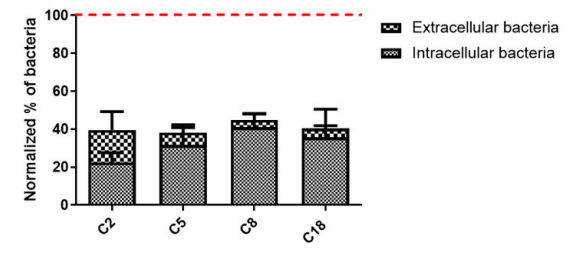

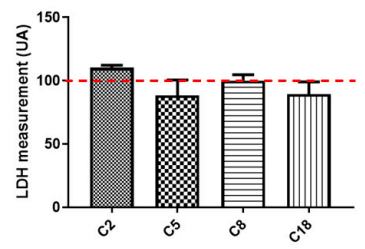

Figure 3. Fate of the internalized commensal C. acnes. (a) Stability of biofilm increase for intracellular C. acnes strains. Each time, red histograms represent the quantification of the initial biofilm formed before internalization. Other histograms have a number corresponding to the number of cultures after internalization $(\$, p<0.05)$; (b) percentage of viable intracellular and extracellular bacteria following $48 \mathrm{~h}$ post-infection; $100 \%$ corresponding to intracellular $C$. acnes strains at $3 \mathrm{~h}$ of interaction (red dot line); (c) LDH release measurement normalized on cells without bacteria, in supernatant after $48 \mathrm{~h}$ of $C$. acnes/osteoblast-like cells interaction; $100 \%$ corresponding to the measurement of cells without bacteria.

\subsection{Behaviors Comparison with BPI C. acnes Strains}

C. acnes strains isolated from BPIs (shoulder prosthesis or osteosynthesis material infection) were studied for their capacity to initiate biofilms, to be internalized by osteoblast-like cells and to form biofilm post-internalization (Figure 4a,b). We have not noticed a significant difference in planktonic growth for all the tested strains before and after internalization by osteoblast-like cells (Figure S1), with the exception of BPI8.

(a)

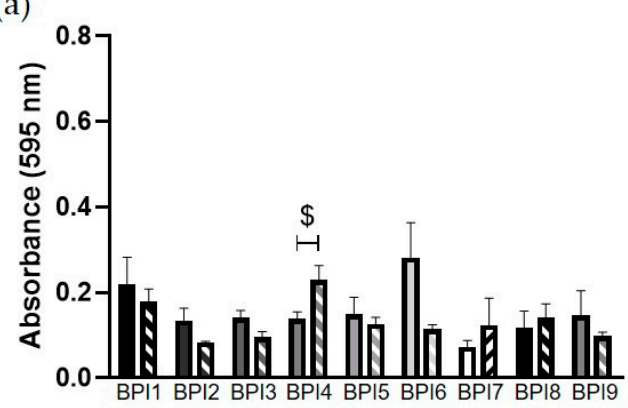

(b)

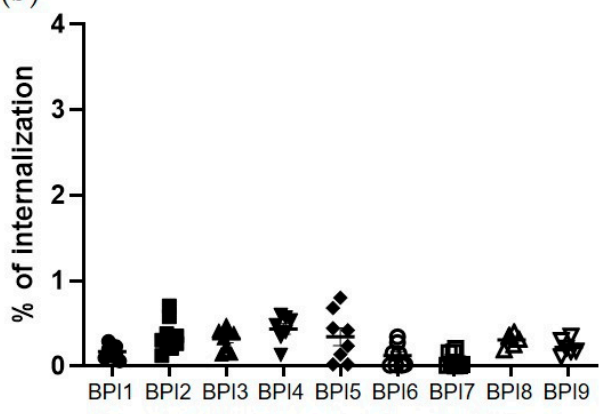

(c)

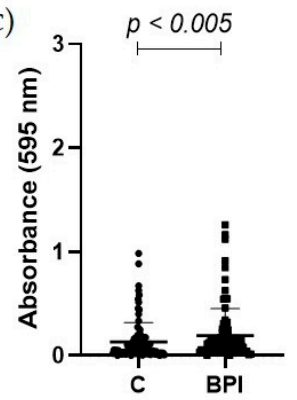

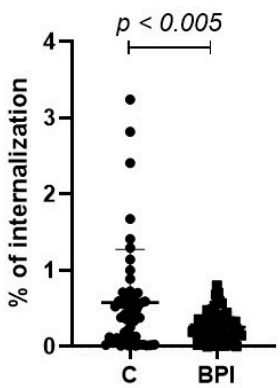

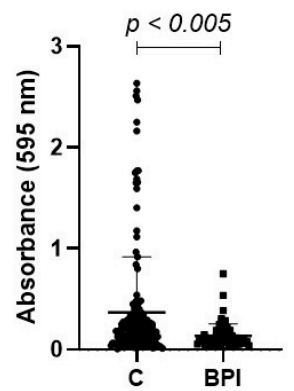

Figure 4. Behaviors of the $C$. acnes BPI strains and comparison with the commensal strains. (a) Biofilm forming capacity by crystal violet quantification without interaction (full bars) and after internalization (hatched bars) by osteoblast-like cells by crystal violet quantification $\$ ; p<0.05$ compared to initial biofilm quantification; (b) internalization rate (\%) by osteoblast-like cells; (c) comparison commensal and BPI strains compiled results: behaviors of $C$. acnes commensal " $\mathrm{C}$ " strains and strains isolated from BPIs: biofilm quantification by crystal violet assay (left graph); internalization rate (\%) by osteoblast-like cells (middle graph); and biofilm quantification after internalization (right graph). 
Figure 4c illustrates the comparison between the commensal and BPI C. acnes strains. As for the commensal strains, the BPI strain genotype was determined by the MLST approach. Six BPI strains were identified with an IA1 phylotype: three were isolated from shoulders, two from the spine and one from the hip (Table 1). Three strains presented an IB phylotype: two of them were isolated from shoulder prostheses and one from tibia material.

After 5 days of biofilm development, we observed an absorbance average of $0.192 \pm 0.03$ with a lower value of 0.07 for BPI 7 and the highest value of 0.28 for BPI 6 (Figure $4 \mathrm{a}$ ). All the BPI strains possessed a similar internalization rate with an average of $0.26 \pm 0.02 \%$ (Figure $4 \mathrm{~b}$ ). Concerning the formed biofilm by intracellular bacteria (Figure $4 \mathrm{a}$ ), no significant change was observed at the exception of strain BPI 4, which showed an increase in biofilm formation post-internalization (1.64-fold; $p<0.05$ ). Nevertheless, in order to facilitate the comparison between the strains from a different origin of isolation (commensal "C" vs. BPI stains), the presented data were compiled (Figure 4c). Thus, the difference in behavior between the strains was highlighted in particular for the initial biofilm formation, which was slightly weaker for the commensal strains $(0.13 \pm 0.02)$ compared to the BPI strains $(0.192 \pm 0.03$; $p<0.05)$. In addition, we noticed a significant difference with a higher internalization rate for the commensal strains compared to the BPIs ( $0.58 \pm 0.1$ vs. $0.26 \pm 0.02$, respectively; $p<0.05)$, while the data distribution was also wider for the commensal strains. Then, the commensal bacteria after internalization formed more biofilm than the BPI strains $(0.37 \pm 0.04$ vs. $0.31 \pm 0.01$, respectively; $p<0.05$ ) with very high values (absorbance of 3.0).

Concerning the live/dead proportion of bacteria within the biofilm (Figure 5a,b), each BPI strain had an increased proportion of live bacteria after internalization, except for BPI2. Figure 5c illustrated a mean proportion of $61.2 \%$ of BPI live bacteria before internalization (values from 41 to $82 \%$ according to the strains), which increased to $76.2 \%$ after internalization (values from 62 to $97 \%$ ), $p<0.05$. However, even if the proportion of live/dead bacteria is similar for all strains before and after internalization (Figure 5c), an increase is observed in the proportion of live bacteria of $25 \%$ for BPIs and $60 \%$ for commensal strains within the formed biofilm post-internalization $(p<0.05)$.
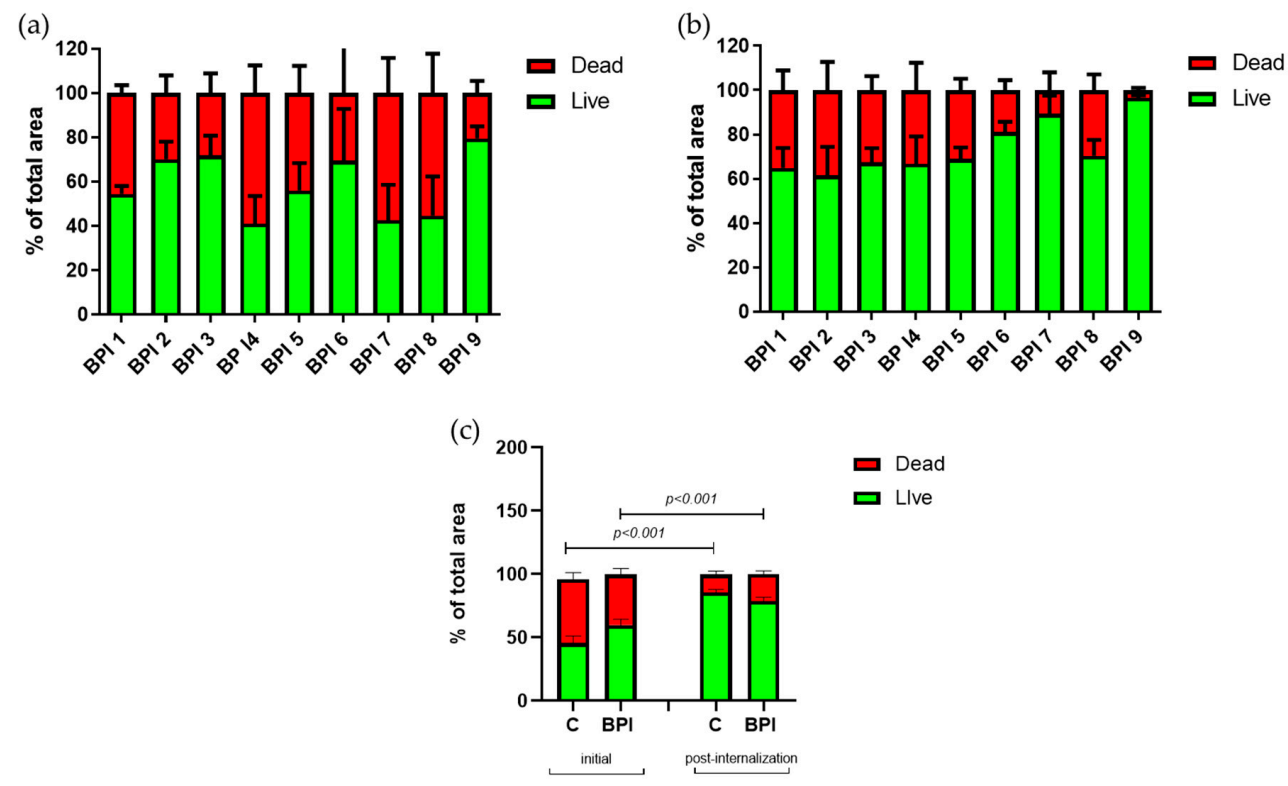

Figure 5. The living bacterial proportion in the BPI C. acnes strain biofilms and comparison with the commensal strain biofilms. Repartition of Syto9 (green bars) and PI (red bars) staining within biofilm formed by the C. acnes strains isolated from BPIs before (a) and after internalization (b) by osteoblast-like cells; (c) comparison of commensal and BPI strains: live dead proportion quantification within biofilm of $C$. acnes commensal " $C$ " strains and isolated from BPIs. Images acquisition by fluorescent microscopy and calculation by Image J software. 


\subsection{Towards In Vivo (Commensal Biofilm on Titanium)}

The biofilm formation of the four-selected commensal C. acnes strains on titanium was studied, as the main material used for the manufacturing of prostheses. We compared the adhesion of the commensal strains on plastic and on titanium (Figure 6). The biofilm-forming ability of our commensal strains is much higher on titanium surfaces than plastic regardless of interaction with SaOS2 cells. A 22- and 21.6-fold increase were observed for the formed biofilm on titanium vs. plastic, respectively, before and after internalization (Figure 6).

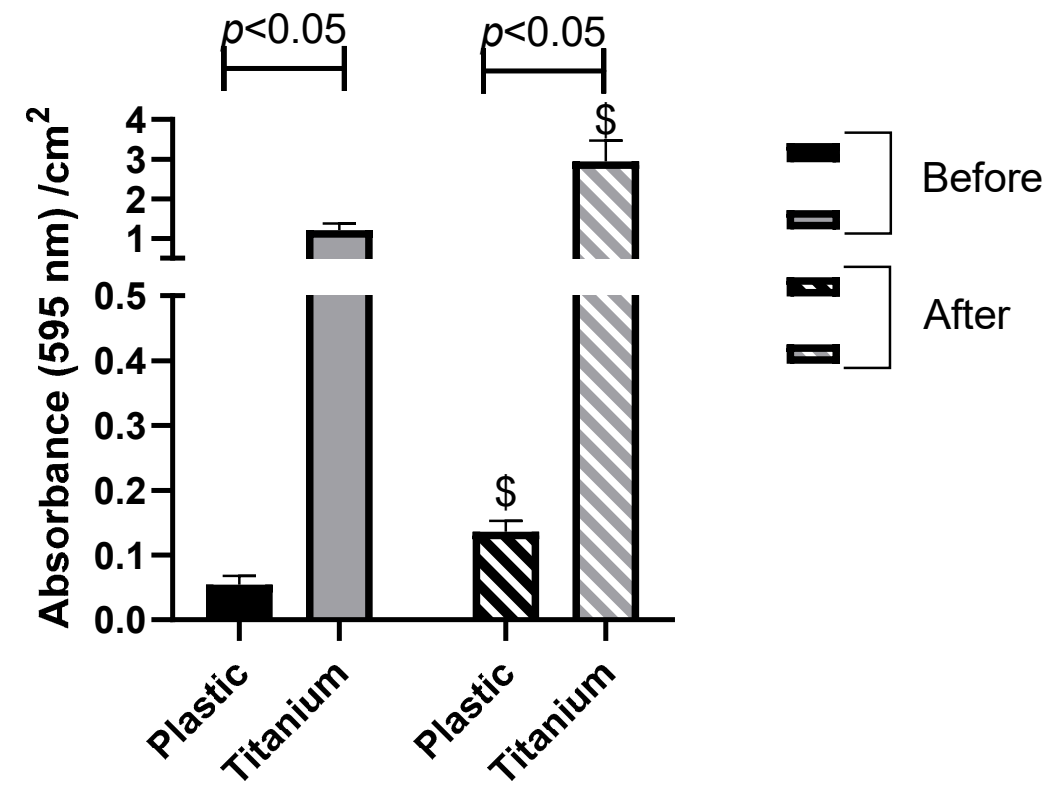

Figure 6. Comparison of the commensal strains' adhesion (compiled results of the four strains) between plastic and titanium surfaces before and after internalization by osteoblast-like cells. Biofilm-forming capacity by crystal violet quantification $(\$$, significant difference with formed biofilm before internalization, $p<0.01)$.

\section{Discussion}

In recent years, Cutibacterium acnes has been increasingly reported as one of the most frequently isolated pathogens in deep tissue infection, complicating shoulder surgery [2,21]. This commensal microbiota can somehow induce BPIs distant-time to the implantation surgery. The objective of this work was to evaluate the virulence of naive C. acnes strains (from commensal flora) in contact with bone cells to understand the physiopathology of these infections. For the first time, to our knowledge, we showed that the commensal bacterial internalization by osteoblast-like cells had an impact on their biofilm formation capacity and this effect was even amplified on a titanium support.

We showed that $C$. acnes was internalized by osteoblast-like cells (SaoS2), regardless of the clinical origin (commensal or BPIs). Some studies have already demonstrated how C. acnes can be internalized into cell lines, such as the prostate epithelial cell line (RWPE-1) [22] or human acute monocytic leukemia cell line THP-1 [23]. Our results are in accordance with Aubin et al., who revealed that C. acnes is also internalized by another bone cell line, MG63, and this phenomenon is not correlated to the clinical origin of the strains [24].

In this study, although there is a significant difference in the rate of internalization between commensal and BPIs, the overall rate of internalization by osteoblast-like cells is less than $1 \%$, regardless of the clinical origin, in below the $30 \%$ internalization rate found in S. aureus [8].

However, we wondered if the commensal strains could persist or exit from the osteoblast-like cells after internalization. After interaction during $3 \mathrm{~h}$ and elimination of the extracellular bacteria, the internalized bacteria's fate was analyzed in extending the culture of the infected cells for $48 \mathrm{~h}$. We did 
not notice any cytotoxic effect on the infected osteoblast-like cells, contrary to S. aureus infections [19]. A minority part of $C$. acnes became extracellular $(8.5 \pm 2.9 \%)$, probably due to the mechanisms of the bone cells. More than $50 \%$ of the internalized bacteria were not found after $48 \mathrm{~h}$, strongly assuming a high death rate $(61.5 \%)$. One tierce of bacteria remained intracellular $(32.7 \pm 3.9 \%)$. This phenomenon of intracellular persistence may allow $C$. acnes to survive in a quiescent form that can be reactivated and proliferate at the latent infection site [23]. This intracellular survival, even a temporary one, allowed the selection of the most resistant bacteria and could have consequences on bacterial behavior and especially on their virulence.

Another important step for bacterial virulence is biofilm formation. Biofilms can be initiated in two different situations: directly when the bacteria get in contact with biotic or abiotic support or indirectly once the bacteria are released from their hosting cell after their first internalization. The main goal of the current study is the comparison of biofilm formation before and after internalization. In our experiments, all strains formed biofilm even if the commensal strains initially formed less biofilm biomass than the BPI strains, confirming previous results [12]. Interestingly, the commensal strains developed more biofilm after internalization with a very high value. In detail, the intracellular bacteria of three skin strains of six formed a significant higher biofilm and one more strain tended to possess the same capacity. On the contrary, the BPI strains formed equal amounts of biofilm pre- and post-internalization, except for strain BPI 4.

The more robust biofilms formed by the four commensal strains seemed to be a non-common phenomenon as two of the four strains kept the phenotype after culture and subcultures, but with lower values, which underlines the possibility of an adaptive and non-hereditary response. As observed, the internalization had no impact on the antibiotic sensibility of the strains, which supported the hypothesis of a temporary change.

Using fluorescent staining, an increase in the live bacteria proportion within the biofilm after internalization was observed regardless of the isolates' origins. These results suggested that the bacterial stress felt during internalization could lead to a stress adaptation and a better survival of bacteria within the biofilm. This could enhance the biofilm mechanisms in the commensal strains already set up in the BPI strains [10]. C. acnes within biofilms presents upregulated, stress-induced genes and CAMP virulent factors involved in host colonization [11,25]. This finding could explain why the biofilm capacity is increased in commensal strains post-internalization. We supposed that the matrix englobing the bacteria could have a different composition after internalization due to the ignition of a "biofilm" program [10]. It would be interesting to quantify the extracellular DNA, proteins and polysaccharides, which compose the biofilm matrix pre- and post-internalization [26].

Finally, in the clinical context, C. acnes adhere to prostheses, mostly made of titanium. Looking at the adhesion of the commensal bacteria on titanium, the biofilm formation was greatly increased on the titanium support as compared to the plastic one. Once again, more biofilm was formed with bacteria that are intracellular. This showed that C. acnes is strongly attracted to titanium, underlining the clinical results.

No link was identified between the MLST profiles and internalization rates or biofilm behavior. However, two BPI strains (BPI8 and BPI9) belonged to the phylotype IB CC5, whereas this phylotype was not identified in our commensal strains panel. This finding corroborated previous data published by Aubin et al. on the high frequency of this phylotype in infective strains (corresponding to IB CC36 phylotype in Aubin et al., with the MLST9 detection profile [27].

Further investigations will be necessary to decipher the mechanism of interaction between the bone cells and bacteria. It might be supported by a conservative motif, such as the fibronectin-binding protein necessary for internalization of $S$. aureus $[8,28]$, with a different level of expression according to the strain and its microenvironment without a genetic background link [29].

It will be interesting to understand the modifications undergone by the bacteria during internalization (affect metabolism, stress proteins, etc.). Indeed, genetic modification or a punctual mutation could modify the expression of adhesins and so the strain pathogenicity, leading to the 
transformation of commensal C. acnes into pathogens [30]. Therefore, interleukins and chemokines production, reflecting inflammatory processes, will have to be measured to comprehend the bone cells response [21]. These findings are essential to enhance the prevention and the management of the orthopedic implant-associated infections. It is of great importance to gain comprehensive knowledge of the infection pathogenesis thanks to this new approach of research study.

\section{Conclusions}

The commensal C. acnes strains' internalization by osteoblast-like cells had an impact on their biofilm formation capacity, unlike the BPI strains. This effect was amplified on a titanium support. Moreover, all the strains, after internalization, showed an increase in the live proportion within biofilms.

Supplementary Materials: The following are available online at http://www.mdpi.com/2076-2607/8/9/1409/s1, Figure S1: Planktonic growth of C. acnes commensal strains, Figure S2: Cytotoxicity after $3 \mathrm{H}$ of $C$. acnes/ osteoblast-like cells interaction.

Author Contributions: F.R. and C.M. conceived and designed the experiments, analyzed the data and wrote this article; J.V.-S., F.L., T.S.E.-M., C.M. and F.R. performed the experiments; X.O., V.V.-G. and L.B. performed all clinical analysis on the isolated strains; S.C.G. supervised the C. acnes experiments. All authors have read and agreed to the published version of the manuscript.

Funding: We acknowledge the University Hospital of Reims for financial support (AOL CUDIOA). TEM is supported by a post-doctoral grant from the Egyptian Science and technology development fund (STDF)/French Institute of Egypt (IFE) and by the Region Grand-Est. F.L.'s PhD is supported by the Region Grand-Est and Foundation URCA.

Acknowledgments: We thank Emilie Charpentier and Janick Madoux for technical support and discussion. Frédéric Velard of the PICT-URCA platform is also thanked for technical assistance.

Conflicts of Interest: The authors declare no conflict of interest.

\section{References}

1. Hsu, J.E.; Bumgarner, R.E.; Matsen, F.A. Propionibacterium in Shoulder Arthroplasty: What We Think We Know Today. J. Bone Joint Surg. Am. 2016, 98, 597-606. [CrossRef]

2. Boisrenoult, P. Cutibacterium acnes prosthetic joint infection: Diagnosis and treatment. Orthop. Traumatol. Surg. Res 2018, 104, S19-S24. [CrossRef] [PubMed]

3. Anagnostopoulos, A.; Bossard, D.A.; Ledergerber, B.; Zingg, P.O.; Zinkernagel, A.S.; Gerber, C.; Achermann, Y. Perioperative Antibiotic Prophylaxis Has No Effect on Time to Positivity and Proportion of Positive Samples: A Cohort Study of 64 Cutibacterium acnes Bone and Joint Infections. J Clin. Microbiol. 2018, 56, e01576-17. [CrossRef] [PubMed]

4. Liew-Littorin, C.; Brüggemann, H.; Davidsson, S.; Nilsdotter-Augustinsson, Å.; Hellmark, B.; Söderquist, B. Clonal diversity of Cutibacterium acnes (formerly Propionibacterium acnes) in prosthetic joint infections. Anaerobe 2019, 59, 54-60. [CrossRef] [PubMed]

5. Pauzenberger, L.; Heller, V.; Ostermann, R.C.; Laky, B.; Heuberer, P.R.; Anderl, W. Cutibacterium Acnes (Formerly Propionibacterium Acnes) Contamination of the Surgical Field During Shoulder Arthroscopy. Arthroscopy 2019, 35, 1750-1757. [CrossRef]

6. Coenye, T.; Peeters, E.; Nelis, H.J. Biofilm formation by Propionibacterium acnes is associated with increased resistance to antimicrobial agents and increased production of putative virulence factors. Res. Microbiol. 2007, 158, 386-392. [CrossRef] [PubMed]

7. Aubin, G.G.; Baud'huin, M.; Lavigne, J.P.; Brion, R.; Gouin, F.; Lepelletier, D.; Jacqueline, C.; Heymann, D.; Asehnoune, K.; Corvec, S. Interaction of Cutibacterium (formerly Propionibacterium) acnes with bone cells: A step toward understanding bone and joint infection development. Sci. Rep. 2017, 7, 42918. [CrossRef]

8. Josse, J.; Laurent, F.; Diot, A. Staphylococcal adhesion and host cell invasion: Fibronecting binding and other mechanisms. Front. Microbiol. 2017, 8, 2433. [CrossRef]

9. Achermann, Y.; Goldstein, E.J.C.; Coenye, T.; Shirtliff, M.E. Propionibacterium acnes: From commensal to opportunistic biofilm-associated implant pathogen. Clin. Microbiol. Rev. 2014, 27, 419-440. [CrossRef] 
10. de laFuente-Núñez, C.; Reffuveille, F.; Fernández, L.; Hancock, R.E. Bacterial biofilm development as a multicellular adaptation: Antibiotic resistance and new therapeutic strategies. Curr. Opin. Microbiol. 2013, 16, 580-589. [CrossRef]

11. Dréno, B.; Pécastaings, S.; Corvec, S.; Veraldi, S.; Khammari, A.; Roques, C. Cutibacterium acnes (Propionibacterium acnes) and acne vulgaris: A brief look at the latest updates. J. Eur. Acad. Dermatol. Venereol. 2018, 32 (Suppl. 2), 5-14. [CrossRef]

12. Holmberg, A.; Lood, R.; Mörgelin, M.; Söderquist, B.; Holst, E.; Collin, M.; Christensson, B.; Rasmussen, M. Biofilm formation by Propionibacterium acnes is a characteristic of invasive isolates. Clin. Microbiol. Infect. 2009, 15, 787-795. [CrossRef] [PubMed]

13. McDowell, A.; Nagy, I.; Magyari, M.; Barnard, E.; Patrick, S. The opportunistic pathogen Propionibacterium acnes: Insights into typing, human disease, clonal diversification and CAMP factor evolution. PLoS ONE 2013, 8, e70897. [CrossRef] [PubMed]

14. Corvec, S.; Dagnelie, M.A.; Khammari, A.; Dréno, B. Taxonomy and phylogeny of Cutibacterium (formerly Propionibacterium) acnes in inflammatory skin diseases. Ann. Dermatol. Venereol. 2019, 146, 26-30. [CrossRef]

15. Paugam, C.; Corvec, S.; Saint-Jean, M.; Le Moigne, M.; Khammari, A.; Boisrobert, A.; Nguyen, J.M.; Gaultier, A.; Dréno, B. Propionibacterium acnes phylotypes and acne severity: An observational prospective study. J. Eur. Acad. Dermatol. Venereol. 2017, 31, e398-e399. [CrossRef] [PubMed]

16. Kilian, M.; Scholz, C.F.; Lomholt, H.B. Multilocus sequence typing and phylogenetic analysis of Propionibacterium acnes. J. Clin. Microbiol. 2012, 50, 1158-1165. [CrossRef] [PubMed]

17. Dekio, I.; Culak, R.; Fang, M.; Ball, G.; Gharbia, S.; Shah, H.N. Correlation between phylogroups and intracellular proteomes of Propionibacterium acnes and differences in the protein expression profiles between anaerobically and aerobically grown cells. Biomed. Res. Int. 2013, 151797.

18. Osmon, D.R.; Berbari, E.F.; Berendt, A.R.; Lew, D.; Zimmerli, W.; Steckelberg, J.M.; Rao, N.; Hanssen, A.; Wilson, W.R. Infectious Diseases Society of America. Diagnosis and management of prosthetic joint infection: Clinical practice guidelines by the Infectious Diseases Society of America. Clin. Infect. Dis. 2013, 56, e1-e25. [CrossRef] [PubMed]

19. Josse, J.; Guillaume, C.; Bour, C.; Lemaire, F.; Mongaret, C.; Draux, F.; Velard, F.; Gangloff, S.C. Impact of the Maturation of Human Primary Bone-Forming Cells on Their Behavior in Acute or Persistent Staphylococcus aureus Infection Models. Front. Cell Infect. Microbiol. 2016, 6, 64. [CrossRef] [PubMed]

20. Reffuveille, F.; Josse, J.; Velard, F.; Lamret, F.; Varin-Simon, J.; Dubus, M.; Haney, E.F.; Hancock, R.E.; Mongaret, C.; Gangloff, S.C. Bone Environment Influences Irreversible Adhesion of a Methicillin-Susceptible Staphylococcus aureus Strain. Front. Microbiol. 2018, 9, 2865. [CrossRef]

21. Gharamti, A.A.; Kanafani, Z.A. Cutibacterium (formerly Propionibacterium) acnes infections associated with implantable devices. Expert. Rev. Anti. Infect. Ther. 2017, 15, 1083-1094. [CrossRef] [PubMed]

22. Mak, T.N.; Fischer, N.; Laube, B.; Brinkmann, V.; Metruccio, M.M.; Sfanos, K.S.; Mollenkopf, H.J.; Meyer, T.F.; Brüggemann, H. Propionibacterium acnes host cell tropism contributes to vimentin-mediated invasion and induction of inflammation. Cell Microbiol. 2012, 14, 1720-1733. [CrossRef] [PubMed]

23. Fischer, N.; Mak, T.N.; Shinohara, D.B.; Sfanos, K.S.; Meyer, T.F.; Brüggemann, H. Deciphering the intracellular fate of Propionibacterium acnes in macrophages. Biomed. Res. Int. 2013, 603046. [CrossRef]

24. Aubin, G.G.; Lavigne, J.P.; Foucher, Y.; Dellière, S.; Lepelletier, D.; Gouin, F.; Corvec, S. Tropism and virulence of Cutibacterium (formerly Propionibacterium) acnes involved in implant-associated infection. Anaerobe 2017, 47, 73-78. [CrossRef]

25. Jahns, A.C.; Eilers, H.; and Alexeyev, O.A. Transcriptomic analysis of Propionibacterium acnes biofilms in vitro. Anaerobe 2016, 42, 111-118. [CrossRef]

26. Flemming, H.-C.; Wingender, J. The biofilm matrix. Nat. Rev. Microbiol. 2010, 8, 623-633. [CrossRef]

27. McDowell, A. Over a Decade of recA and tly Gene Sequence Typing of the Skin Bacterium Propionibacterium acnes: What Have We Learnt? Microorganisms 2017, 6, 1. [CrossRef]

28. Yu, J.L.; Månsson, R.; Flock, J.I.; Ljungh, A. Fibronectin binding by Propionibacterium acnes. FEMS Immunol. Med. Microbiol. 1997, 19, 247-253. [CrossRef] 
29. Costerton, J.W.; Lewandowski, Z.; Caldwell, D.E.; Korber, D.R.; Lappin-Scott, H.M. Microbial biofilms. Annu. Rev. Microbiol. 1995, 49, 711-745. [CrossRef]

30. Brzuszkiewicz, E.; Weiner, J.; Wollherr, A.; Thürmer, A.; Hüpeden, J.; Lomholt, H.B.; Kilian, M.; Gottschalk, G.; Daniel, R.; Mollenkopf, H.J.; et al. Comparative genomics and transcriptomics of Propionibacterium acnes. PLoS ONE 2011, 6, e21581. [CrossRef] 